Casos Clínicos

Arch. Esp. Urol. 2010; 63 (4): 309-312

\section{PÓLIPO FIBROEPITELIAL DEL PENE. PRESENTACIÓN DE UN CASO}

Tomás Lázaro Rodríguez Collar, Basily Valdés Estévez, Laura López Marín y Roxana Soranyer Horroutinel Scull.

Unidad de Urología Hospital Universitario Dr. "Carlos J. Finlay". Ciudad de la Habana. Cuba.

Resumen.- OBJETIVO: Presentar un nuevo caso de pólipo fibroepitelial del pene y su tratamiento.

MÉTODOS: Se presenta el caso de un paciente de 39 años de edad, que asistió a la consulta por notarse un crecimiento de aspecto verrugoso, en el frenillo del pene que le estaba estenosando el meato uretral dificultándole la micción.
RESULTADOS: Se le realizó la exéresis total de la lesión junto con la circuncisión. El estudio histopatológico informó pólipo fibroepitelial. Tras un año de operado el paciente está asintomático y libre de recurrencias.

CONCLUSIÓN: Se confirma la importancia de tener en consideración, este tipo de lesión en el diagnóstico diferencial de los tumores del pene, pues su aspecto macroscópico semeja una neoformación cancerosa en dicho órgano.

Palabras clave: Tumores del pene. Pólipo fibroepitelial. Tratamiento.

Summary.- OBJETIVE: To report a new case of penile fibro-epithelial polyp and its treatment.

METHODS: A 39 year-old patient who came to the outpatient clinic due to a warted growth he had noticed on the frenulum of prepuce of his penis which was narrowing the urethral meatus and impairing his voiding.

RESULTS: Total exeresis of the lesion and circumcision were performed. The histopathologic study revealed a fibroepithelial polyp. The patient is asymptomatic and without any recurrence.

CONCLUSIONS: The importance of considering this type of lesion in the differential diagnosis of penis tumors is confirmed because its macroscopic aspect seems like a cancerogenic new formation on this organ.

Keywords: Penis tumors. Fibro-epithelial polyp. Treatment.

\section{INTRODUCCIÓN}

Los pólipos fibroepiteliales son tumores benignos de origen mesodérmico. En el tracto urinario son raros, y su localización más frecuente es en el uréter. También se han descrito casos en la pelvis renal, la vejiga y la uretra; $(1,2)$ en esta última se observan con más frecuencia en el verumontánum (3) y, en su inmensa mayoría, en niños de la primera década de la vida (4).

Su presencia en el pene está reportada como bastante infrecuente, señalándose su asociación con los procesos de cicatrización posquirúrgica en niños (5) y, como secuela del uso prolongado del condón catéter, para el tratamiento de la incontinencia urinaria en pacientes adultos $(6,7)$.

Presentamos un nuevo caso de pólipo fibroepitelial del pene en un paciente sin antecedentes urológicos previos, y que fue tratado mediante la exéresis quirúrgica de la lesión más la circuncisión. Luego de un año de operado se mantiene asintomático. 


\section{CASO CLÍNICO}

Paciente de 39 años de edad con antecedentes de buena salud. Asiste a la consulta externa de Urología refiriendo la presencia, en la zona del frenillo del pene, de un tumor de apariencia verrugosa, que le había crecido paulatinamente en los últimos cinco meses y le estaba cerrando el meato uretral. Esta situación comenzó a preocuparle mucho más ya que, el chorro urinario se le dispersaba, haciéndosele dificultoso orinar en posición de pie.

Al examen físico se constató la presencia de una lesión en forma de coliflor, multilobulada, sésil, de color rosado claro y no segregante, que ocupaba el frenillo del pene así como la comisura ventral y los bordes laterales del meato uretral (Figura 1).

Se procedió a la exéresis quirúrgica total del tumor más la circuncisión bajo anestesia local. Se logró despejar el meato uretral, dejándolo libre de lesión, con un calibre apropiado y con buenos resultados estéticos (Figura 2).

Se le colocó sonda uretral Foley $18 \mathrm{Fr}$ la cual se le mantuvo por una semana, y tratamiento antibiótico oral con Ciprofloxacina 500 milígramos cada 12 horas durante 10 días.

El informe histopatológico del tejido resecado fue: Pólipo Fibroepitelial del Pene (Figura 3).

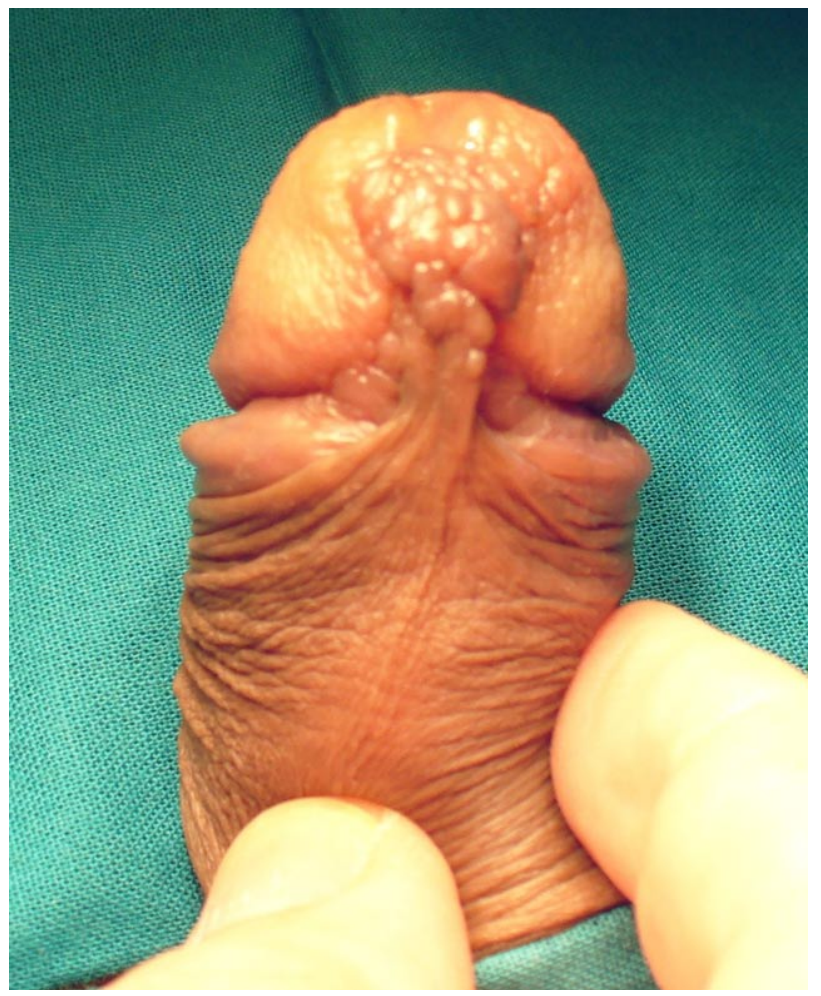

El paciente ha evolucionado satisfactoriamente y después de un año de operado, está totalmente asintomático llevando una vida social y sexual plenas.

\section{DISCUSIÓN}

Los pólipos fibroepiteliales constituyen una variedad de tumores benignos, cuya aparición en los órganos del sistema génito-urinario es infrecuente.

Su ubicación más descrita es en el uréter de pacientes jóvenes que acuden a consulta por hematuria, (8) aunque se han reportado casos totalmente asintomáticos. (9) Otras localizaciones reportadas como la pelvis renal, la vejiga y la uretra son más raras aún y, constituyen hallazgos en piezas quirúrgicas estudiadas por sospecha de cáncer urotelial $(2,8)$. En la uretra posterior de niños debe sospecharse, ante la presencia de infección urinaria recurrente, disuria, hematuria y retención urinaria (4); en los adultos la manifestación clínica más asociada es la hematuria (3).

La presencia en el pene de los pólipos fibroepiteliales es excepcional. En la literatura consultada aparecen dos grupos bien definidos de pacientes en los que estos se desarrollan. Uno de ellos está integrado por niños, los cuales tienen el antecedente de cirugías peneanas. Se invoca que en su patogénesis pueden influir, de manera decisiva, los procesos regenerativos que se producen

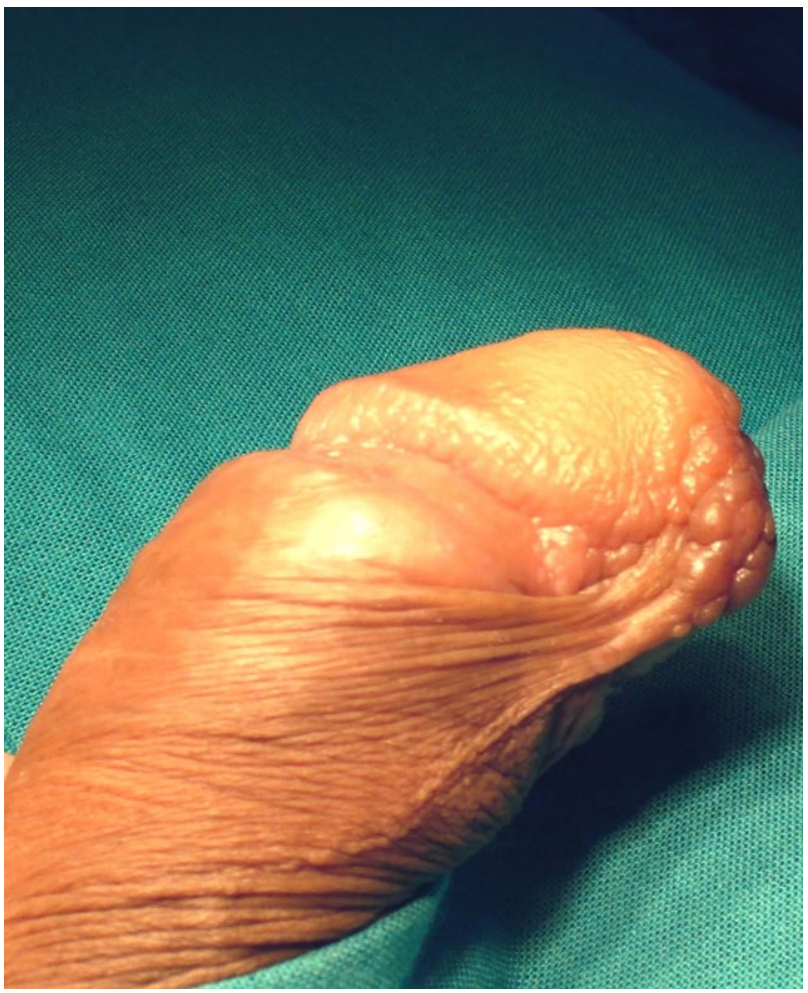

FIGURA 1. Se observa la lesión en forma de coliflor que interesa el frenillo y el meato uretral. 

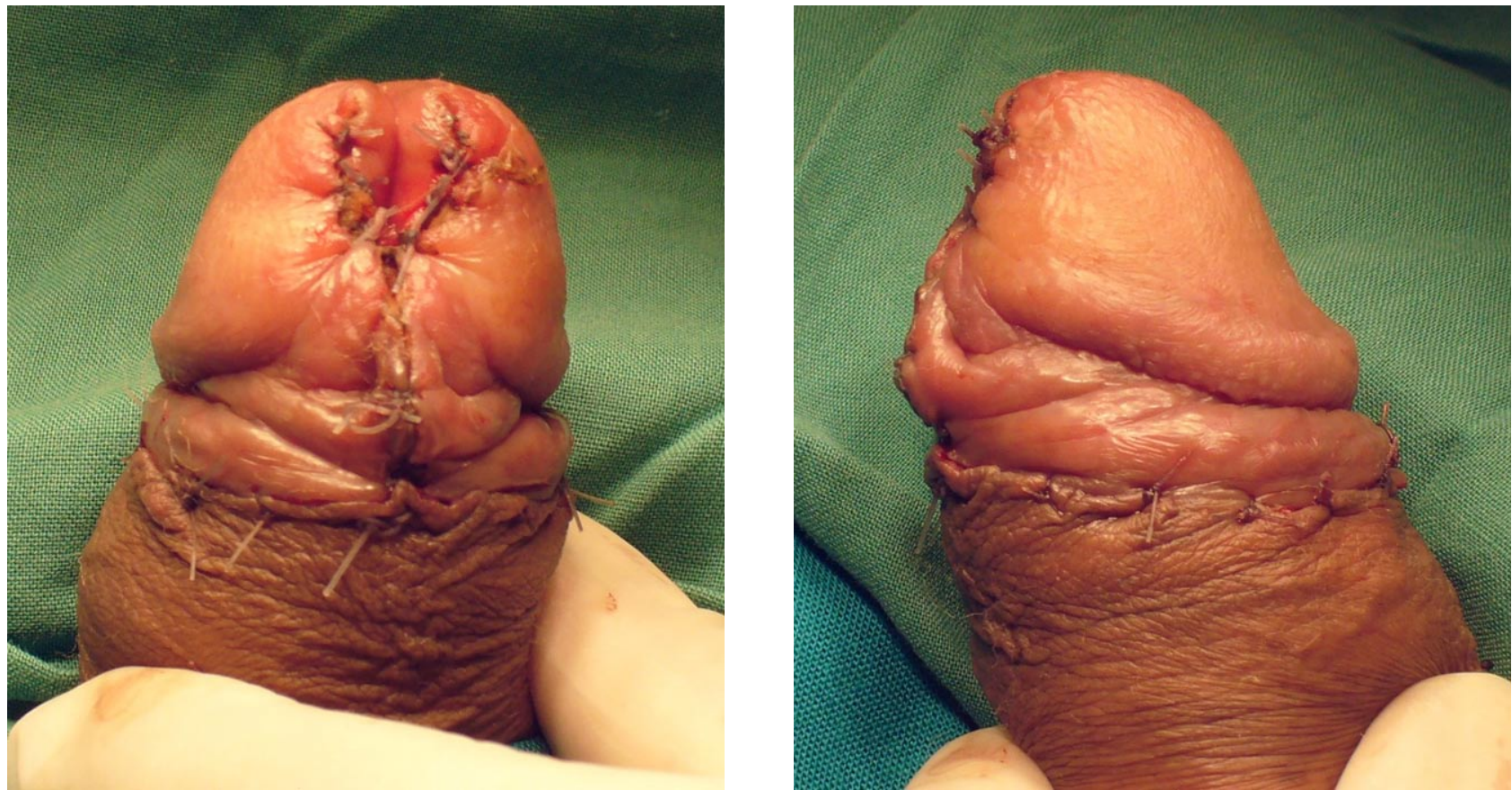

FIGURA 2. Vista posquirúrgica observándose el meato uretral libre de tumor y buen resultado estético final.

durante la cicatrización, los que se tornarían exagerados y favorecerían la hiperplasia del tejido fibroconectivo local $(5,10)$.

El otro grupo lo componen pacientes adultos que usaron, por muchos años, el condón catéter por presentar incontinencia urinaria. En estos casos se plantea que el traumatismo crónico es el agente causal del surgimiento de tales lesiones $(6,7)$.

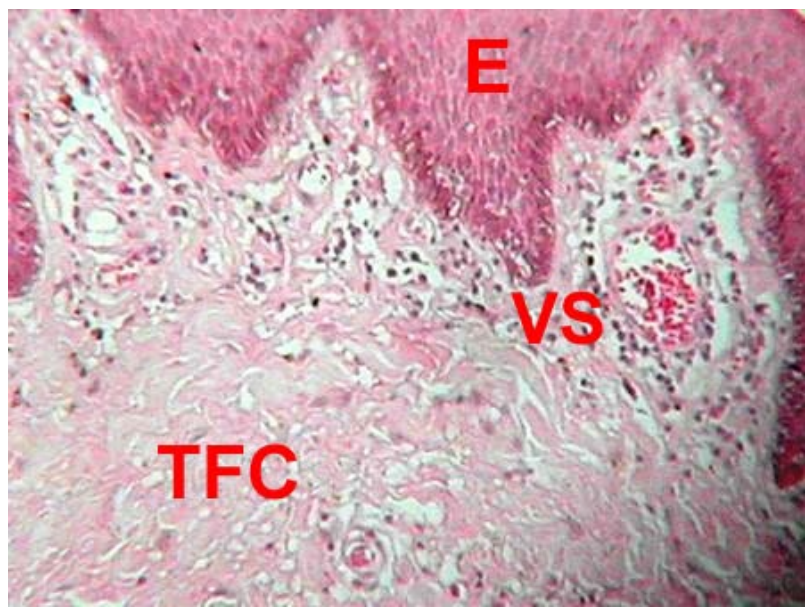

FIGURA 3. Vista microscópica del tumor. Se aprecian la hiperplasia del epitelio (E), los vasos sanguíneos (VS) y el tejido fibroconectivo (TFC). HE X 200.
El caso que presentamos no reúne ninguna de las dos condiciones antes planteadas $y$, durante el interrogatorio exhaustivo realizado, no se logró obtener ningún dato o suceso que pudiera tener relación con la formación del pólipo fibroepitelial del pene en este paciente.

El tratamiento que se sugiere por los autores consultados es el quirúrgico, realizándose la exéresis de la lesión teniendo los pacientes una evolución satisfactoria, no reportándose recidiva tumoral $(5-7,10)$. En nuestro paciente se tomó la misma conducta terapéutica y evolucionó favorablemente.

Desde el punto de vista histológico se describe que el pólipo fibroepitelial presenta, de forma típica, un cono de estroma fibroconectivo vascularizado, cubierto por epitelio con cierto grado de hiperplasia, que según su situación anatómica, puede ser de células transicionales o escamosas $(2,8)$. En el caso que presentamos se encontró este mismo patrón en el estudio microscópico, acompañado de hiperplasia epitelial escamosa.

\section{CONCLUSIÓN}

Como conclusión de este trabajo, debemos pensar en la posibilidad de la existencia del pólipo fibroepitelial, cuando estemos en presencia de un fumor del pene, ya que este forma parte de las entidades nosológicas que pudieran semejar, por su aspecto macroscópico, una lesión cancerosa en dicho órgano. 


\section{BIBLIOGRAFIA y LECTURAS RECOMENDADAS (*lectura de interés $y^{* *}$ lectura fundamental)}

1. Kumar A, Das SK, Trivedi S, Dwivedi US, Singh PB. Genito-urinary polyps: summary of the 10 -year experiences of a single institute. Int Urol Nephrol. 2008; 40: 901-7.

2. Epstein JI. The lower urinary tract and male genital system. In: Kumar V, Abbas AK, Fausto N, editors. Robbins and Cotran Pathologic Basis of Disease. 7th ed. Saunders, Philadelphia. 2005.p.1025.

3. Lanzas Prieto JM, Menéndez Fernández CL, Pérez García FJ, Gutiérrez García R, González Tuero JL, Guate Ortiz JL. Pólipo fibroepitelial de uretra en un adulto. Actas Urol Esp. 2003; 27(8):654-56.

4. Nortes Baños L, Bañón Pérez VJ. Pólipo fibroepitelial de uretra posterior en niños. Actas Urol Esp. 2000; 24 (4):355-57.

*5. Eisner BH, Gargollo PC, Cendron M, Peters CA, Pérez-Atayde A, Diamond DA. Fibroepithelial polyps of the penis in children. Poster. 75th Annual Meeting. New England Section of the American Uro- logical Association. September 2006. http://www. NEAUA.org

**6. Al- Awadhi NM, Al- Brahim N, Ahmad MS, Yordanov E. Giant fibroepithelial polyp of the penis associated with long - term use of condom catheter. Case report and literature review. Can J Urol. 2007 Aug; 14 (4): 3656-9.

*7. Turgut M, Yenilmez A, Can C, Bildirici K, Erkul A, Özyürek Y. Fibroepithelial polyps of glans penis. J Urol. 2005 Mar; 65 (3): 593.

8. Gupta M, Ost MO, Shah JB, Mc Dougall EM, Smith AD. Percutaneous management of the upper urinary tract. In: Wein AJ, Kavoussi LR, Novick AC, Partin AW, Peters CA, editors. Campbell - Walsh Urology. 9th ed. [CD-ROM ]. Saunders Elsevier, Philadelphia. 2007.

9. Morales R, Manrique E, Casanova R, Molina P, Falcón R. Fibroepithelial polyp of the ureter. Report of one case. Arch Urol Esp. 2004 May;57(4):440-42.

**10. Yildirim I, Irkilata C, Sumer F, Aydur E, Ozcan A, Dayanc M. Fibroepithelial polyp originating from the glans penis in a child. Int J Urol. 2004 Mar; 11(3):187-8. 